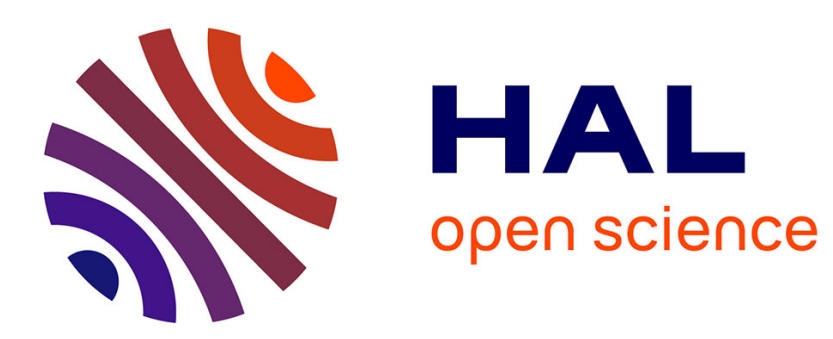

\title{
Generic Delay-L Left Invertibility of Structured Systems with Scalar Unknown Input
}

Federica Garin, Alain Kibangou

\section{To cite this version:}

Federica Garin, Alain Kibangou. Generic Delay-L Left Invertibility of Structured Systems with Scalar Unknown Input. CDC 2019 - 58th IEEE Conference on Decision and Control, Dec 2019, Nice, France. hal-02307596

\section{HAL Id: hal-02307596 https://hal.inria.fr/hal-02307596}

Submitted on 7 Oct 2019

HAL is a multi-disciplinary open access archive for the deposit and dissemination of scientific research documents, whether they are published or not. The documents may come from teaching and research institutions in France or abroad, or from public or private research centers.
L'archive ouverte pluridisciplinaire $\mathbf{H A L}$, est destinée au dépôt et à la diffusion de documents scientifiques de niveau recherche, publiés ou non, émanant des établissements d'enseignement et de recherche français ou étrangers, des laboratoires publics ou privés. 


\title{
Generic Delay-L Left Invertibility of Structured Systems with Scalar Unknown Input.
}

\author{
Federica Garin and Alain Y. Kibangou
}

\begin{abstract}
This paper concerns structured systems, namely linear systems where the state-space matrices have zeros in some fixed positions, and free parameters in all other entries. In particular, it focuses on discrete-time linear time-invariant systems affected by an unknown input. The goal is to study delay- $L$ left invertibility, namely the possibility to reconstruct the input sequence from the output sequence, assuming that the initial state is known, and requiring that the inputs can be reconstructed at least up to $L$ time steps before the current output. Under the assumption that the unknown input is scalar, this paper presents a simple graphical condition characterizing the structured systems which are generically delay- $L$ left invertible.
\end{abstract}

\section{INTRODUCTION}

The study of network systems has given rise to great recent attention towards the so-called structured systems, where a given pattern of zeros is imposed to the matrices in the state-space realization of a system, representing known lack of interaction between some states, while all other entries are free parameters. The zero pattern can be equivalently described with a directed graph, where edges represent nonzero entries. The goal is to find graphical conditions ensuring that some system-theoretic property is true generically, where generically (or structurally, or for almost all parameters) means for all parameters except possibly those lying in a proper subvariety of the parameter space. This also has a probabilistic interpretation: since a proper subvariety has zero Lebsegue measure, a property which is generically true is also true with probability 1 , if the parameters are chosen at random, according to any continuous distribution.

The most classical results on structured systems concerned controllability and observability, with the seminal paper by Lin [1] and a rich literature well summarized in the book [2] and in the survey paper [3], which include strucutral results on many other system-theoretic properties.

This paper focuses on structured systems with an unknown input. This may represent a fault, an unmodeled part of the system, or a malicious external attack. The latter interpretation has brought significant attention to systems with an unknown input in the research area of cyber-physical security. In particular, papers [4] and [5] study perfect attacks, namely attacks that can go completely undetected, since they produce the same output as a legitimate trajectory without input; extensions to near-perfect attacks are proposed in [6], to include attacks producing small albeit non-zero residuals in the attack detector. In [4], assuming that the initial state

The authors are with Univ. Grenoble Alpes, Inria, CNRS, Grenoble INP, GIPSA-lab, Grenoble, France, federica.garin@inria.fr, alain.kibangoudujf-grenoble.fr is known, the authors characterize perfect attackability as the lack of left invertibility, and then they study generic left invertibility of structured systems, including an extension to descriptor systems. Instead, [5] focuses on the optimization problem related to sensor placement, where the goal is to ensure left invertibility with the smallest number of dedicated sensors, each measuring one local state, and furthermore minimizing the communication between sensors.

When the initial state is not perfectly known (and hence also in the presence of noise, when one would like to use a Luenberger-like observer or a Kalman-like filter, suitably adapted to deal with the unknown input), the notion of left invertibility should be accompained by the one of strong observability, namely the possibility to reconstruct the initial state from the output measurements, despite the presence of the unknown input. Strong observability and left invertibility together have been studied under the name of state-and-input observability or input-and-state observability. This property has been characterized for structured systems in [7]; see also [8] and [9] for a simpler rephrasing of their characterization and for further results, in [8] about optimal sensor placement, and in [9] about the additional property of delay-1 left invertibility, discussed below.

Above-mentioned works (except [9]) do not take into account the delay of the left invertibility: for which $L$ can we reconstruct inputs $u(0), \ldots, u(t-L)$ from the initial state $x(0)$ and measurements $y(0), \ldots, y(t)$ ?

This notion of delay is crucial when one implements observers for recursively reconstructing the input and the state, as highlighted in [10]. Classical studies by Massey and Sain have found an algebraic condition characterizing delay- $L$ left invertibility as the rank of a matrix involving the matrices of the state-space representation of the system [11]. Also see [12] for a thourough discussion of the counterpart of delay- $L$ left invertibility for continuous-time systems.

Results about generic delay- $L$ left invertibility have only focused on delay 1, with a characterization in [9] for linear time-invariant systems. A series of papers summarized in the thesis [13] have studied various aspects of delay-1 left invertibility together with strong observability, including time-varying systems and strongly structural results, where graphical conditions ensure that a property is true for all non-zero parameters instead of generically.

The novelty of this paper is the study of generic delay$L$ left invertibility for structured systems, for any given delay $L$. Our main result is restricted to systems with scalar input, for which we find a simple graphical condition which characterizes generic delay- $L$ left invertibility. This result 


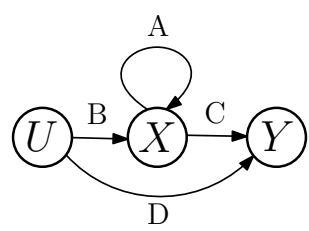

Fig. 1. Pictorial reminder of the construction of the digraph $\mathcal{G}$ of a structured system.

can also be used in combination with the characterization of input-and-state observability [7], [8], [9], to obtain the conditions under which the system can generically have a delay- $L$ observer as in [10].

Our result crucially relies on the assumption that the input is scalar. For the case where the input is not scalar, we present an overview of the partial results in the literature and a counterexample to a natural naive conjecture.

\section{Problem Setup and Preliminaries}

\section{A. Structured System and its Digraph}

Consider a discrete-time linear time-invariant (LTI) system

$$
\left\{\begin{array}{l}
x(t+1)=A x(t)+B u(t) \\
y(t)=C x(t)+D u(t)
\end{array}\right.
$$

where $x(t) \in \mathbb{R}^{n}$ is the state, $u(t) \in \mathbb{R}^{p}$ is the unknown input, and $y(t) \in \mathbb{R}^{m}$ is the output.

Assume matrices $A, B, C, D$ of this state-space representation of the system have fixed zeros in some positions and free parameters in all other positions, namely all entries not fixed to zero are distinct real-valued parameters, that can be chosen arbitrarily, with no constraint relating some parameters to some others. For a given pattern of zeros, the above-described family of systems is known as a structured system. The parameter space is $\mathbb{R}^{\mu}$, where $\mu$ is the total number of entries of the four matrices that are not fixed to zero.

A structured system is uniquely defined by describing the position of zeros in the four matrices. A classical equivalent way of describing a structured system is the following directed graph (digraph), where free parameters are associated with edges (see e.g. [3]). Define the vertex set $U \cup X \cup Y$, where $U:=\left\{u_{1}, \ldots, u_{p}\right\}, X:=\left\{x_{1}, \ldots, x_{n}\right\}$, and $Y:=\left\{y_{1}, \ldots, y_{m}\right\}$ are the input, state and output vertex sets, respectively. The edges correspond to the $\mu$ entries of $A, B, C$, and $D$ that are not fixed to zero, i.e., that contain a free parameter. More precisely, there is:

- an edge $\left(x_{j}, x_{i}\right)$ if and only if $A_{i j}$ is not fixed to 0 ,

- an edge $\left(u_{j}, x_{i}\right)$ if and only if $B_{i j}$ is not fixed to 0 ,

- an edge $\left(x_{j}, y_{i}\right)$ if and only if $C_{i j}$ is not fixed to 0 , and

- an edge $\left(u_{j}, y_{i}\right)$ if and only if $D_{i j}$ is not fixed to 0 .

Figure 1 illustrates this construction, while examples are presented in Figures 2 and 3.

\section{B. Delay-L Left Invertibility}

Here we recall the definition and the classical algebraic characterization of delay- $L$ left invertibility for discrete-time
LTI systems, from [11]; see [12] for the continuous-time interpretation of the same.

Definition 1: For an integer $L \geq 0$, the system (1) is delay-L left invertible if the unknown input $u(0)$ is uniquely determined by the initial state $x(0)$ and the output sequence $y(0), \ldots, y(L)$.

Definition 2: The system (1) is left invertible if there exists an $L$ for which it is delay- $L$ left invertible.

The characterization of delay- $L$ left invertibility from [11] requires to define matrices $M_{L}$ as follows. Let $M_{0}:=D$ and, for $L \geq 1$, recursively define

$$
M_{L}:=\left[\begin{array}{cc}
D & 0 \\
O_{L} B & M_{L-1}
\end{array}\right], \text { where } O_{L}:=\left[\begin{array}{c}
C \\
C A \\
\vdots \\
C A^{L-1}
\end{array}\right]
$$

i.e., $O_{L}$ is the Kalman observability matrix from outputs $y(0), \ldots, y(L-1)$. In other words,

$$
M_{L}=\left[\begin{array}{ccccc}
D & 0 & 0 & \ldots & 0 \\
C B & D & 0 & \ldots & 0 \\
C A B & C B & D & & 0 \\
\vdots & \vdots & \vdots & & \vdots \\
C A^{L-1} B & C A^{L-2} B & C A^{L-3} B & \ldots & D
\end{array}\right] .
$$

The key remark is that

$$
M_{L}\left[\begin{array}{c}
u(0) \\
\vdots \\
u(L)
\end{array}\right]=\left[\begin{array}{c}
y(0) \\
\vdots \\
y(L)
\end{array}\right]-O_{L} x(0) .
$$

For this reason, with the above notation, together with the definition $\operatorname{rank} M_{-1}:=0$, delay- $L$ left invertibility is characterized as follows.

Proposition 1 ([11, Thm. 4]): For any integer $L \geq 0$, the system (1) is delay-L left invertible if and only if

$$
\operatorname{rank} M_{L}=p+\operatorname{rank} M_{L-1}
$$

It is worth recalling that if the system is delay- $L$ left invertible, then it is also delay- $H$ left invertible for all $H \geq L[11$, Thm. 4]. The smallest $L$ such that the system is delay- $L$ left invertible is known as the inherent delay of the system. The inherent delay is infinite if the system is not left invertible, and otherwise it is at most $n$ [12, Coroll. 1].

The classical results summarized in this section give an algebraic characterization of delay- $L$ left invertibility of a system; this characterization involves matrices $A, B, C$, $D$. The goal of this paper is to find a purely graphical condition, characterizing generic delay- $L$ left invertibility of a structured system based only on the pattern of zeros described by the digraph $\mathcal{G}$. 


\section{Main Result}

Our main result is the graphical characterization of generic delay- $L$ left invertibility, under the assumption that the input is scalar.

We consider a structured system defined by its digraph $\mathcal{G}$ as in Sect. II-A, and we look for graphical conditions ensuring that the system is generically delay- $L$ left invertible, i.e., is delay- $L$ left invertible for almost all parameters (for all parameters, except possibly those lying on a proper subvariety of the parameter space).

We assume that the input is scalar $(p=1)$, and we denote by $u$ the only input vertex $u_{1}$. We will use the notation $\operatorname{dist}(u, Y):=\min _{y \in Y} \operatorname{dist}(u, y)$, where the distance $\operatorname{dist}(u, y)$ is defined as usual as the length (number of edges) of the shortest path from $u$ to $y$. Our main result is the following.

Theorem 1: Consider a structured system with scalar input, with digraph $\mathcal{G}$.

- If $L<\operatorname{dist}(u, Y)-1$, then the structured system is not delay- $L$ left invertible, for any choice of the parameters.

- If $L \geq \operatorname{dist}(u, Y)-1$, then the structured system is generically delay- $L$ left invertible.

The key ingredient in the proof of Theorem 1 is the following remark, that relates entries of $M_{L}$ to paths in $\mathcal{G}$.

Remark 1: The entries of $M_{L}$ are obtained looking at suitable paths from $u$ to $Y$ in $\mathcal{G}$.

The non-zero entries of $D$ are in one-to-one correspondence with the edges from $u$ to $Y$; such edges are the paths of length 1 from $u$ to $Y$. In particular, this means that $D=0$ if and only if $\operatorname{dist}(u, Y)>1$.

The entries of $C A^{h} B$, instead, are obtained by looking at paths of length $h+2$. More precisely, the $i$-th entry of the vector $C A^{h} B$ is given by the sum over all paths of length $h+2$ from $u$ to $y_{i}$ of the product of parameters associated to edges in the path. Indeed,

$$
\left[C A^{h} B\right]_{i}=\sum_{k_{0}=1}^{n} \ldots \sum_{k_{h}=1}^{n} C_{i k_{h}} A_{k_{h} k_{h-1}} \ldots A_{k_{1} k_{0}} B_{k_{0}}
$$

and clearly each term of the sum is the product of parameters corresponding to edges of the path $u, x_{k_{0}}, \ldots, x_{k_{h}}, y_{i}$ if such path exists, and is zero if such path does not exist.

From Remark 1, it is easy to obtain the following result about $M_{L}$.

Lemma 1: Consider a structured system with scalar input, with digraph $\mathcal{G} . M_{L}=0$ for all $L<\operatorname{dist}(u, Y)-1$.

Proof: $\quad$ Since $M_{L}$ is defined only for $L \geq 0$, if $\operatorname{dist}(u, Y)=1$ we have nothing to prove. Consider the case where $\operatorname{dist}(u, Y)>1$. By definition of $\operatorname{dist}(u, Y)$, there is no path from $u$ to $Y$ of length strictly smaller than $\operatorname{dist}(u, Y)$, and hence by Remark 1 we have $D=0$ and $C A^{h} B=0$ for all $h<\operatorname{dist}(u, Y)-2$.
We are now ready to prove our main result, exploiting the algebraic characterization in Proposition 1.

Proof of Theorem 1: $\quad$ By Remark 1, $\operatorname{dist}(u, Y)=1$ if and only if $D$ has some entry not fixed to zero. First, we consider the case where $\operatorname{dist}(u, Y)=1$. In this case, generically rank $D=1$ and hence the system is generically delay-0 left invertible. This further implies that the system is generically delay- $L$ left invertible for all $L \geq 0$.

Then, we consider the case where $\operatorname{dist}(u, Y)>1$. For all $L<\operatorname{dist}(u, Y)-1$, by Lemma 1 we have $M_{L}=0$. This implies that $\operatorname{rank} M_{L}=0<p+\operatorname{rank} M_{L-1}=1$ and hence the system is not delay- $L$ left invertible.

For $L=\operatorname{dist}(u, Y)-1$, by Lemma 1 we have $M_{H}=0$ for all $H<L$. This implies that $M_{L}=\left[\begin{array}{cc}0 & 0 \\ C A^{L-1} B & 0\end{array}\right]$, and hence $\operatorname{rank} M_{L}=\operatorname{rank} C A^{L-1} B$. Since $L=\operatorname{dist}(u, Y)-1$, there exists at least one path of length $L+1$ from $u$ to some $y_{i} \in Y$. Hence, by Remark 1 , the $i$-th entry of $C A^{L-1} B$ is a non-zero polynomial in the parameters, and is generically non-zero. This implies that the generic rank of $M_{L}$ is 1 , which is equal to $p+\operatorname{rank} M_{L-1}=1\left(\right.$ since $\left.M_{L-1}=0\right)$. This concludes the proof that the system is generically delay- $L$ left invertible for $L=\operatorname{dist}(u, Y)-1$. Then the same is also true for all larger delays.

Remark 2: The statement of Theorem 1 can be equivalently rephrased as follows: if the input is scalar, then

- the structured system is generically delay- $L$ left invertible if and only if $L \geq \operatorname{dist}(u, Y)-1$;

- the structured system is either generically delay- $L$ left invertible, or not delay- $L$ left invertible for any choice of the parameters.

The first item means that a structured system with scalar input has generic inherent delay $\operatorname{dist}(u, Y)-1$. Also notice that this implies that a structured system with scalar input is delay-0 left invertible if and only if there is a direct feedthrough of the input to the output (i.e., $D \neq 0$, which by $\operatorname{Remark} 1$ is equivalent to $\operatorname{dist}(u, Y)=1)$.

As an immediate corollary of Thm. 1 we can also obtain the following characterization of left invertibility.

Corollary 1: Consider a structured system with scalar input, with digraph $\mathcal{G}$. The structured system is generically left invertible if and only if there exists a path from $u$ to $Y$. Moreover, if there is no path from $u$ to $Y$, then the structured system is not left invertible, for any choice of the parameters.

Example 1: Consider the structured system (1) with the following matrices:

$$
\begin{array}{cc}
A=\left[\begin{array}{ccc}
\alpha_{11} & 0 & \alpha_{13} \\
\alpha_{21} & 0 & 0 \\
0 & \alpha_{32} & 0
\end{array}\right], \quad B=\left[\begin{array}{l}
\beta \\
0 \\
0
\end{array}\right], \\
C=\left[\begin{array}{ccc}
0 & \gamma_{12} & 0 \\
0 & \gamma_{22} & \gamma_{23}
\end{array}\right], \quad D=\left[\begin{array}{l}
0 \\
0
\end{array}\right] .
\end{array}
$$

Its digraph $\mathcal{G}$ is shown in Figure 2. 


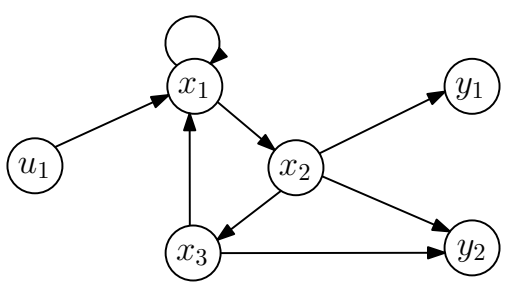

Fig. 2. Digraph $\mathcal{G}$ of the structured system in Example 1.

Notice that $\operatorname{dist}(u, Y)=3$, and hence by Theorem 1 the structured system is not delay- 0 nor delay- 1 left invertible, for any choice of the parameters, and it is generically delay$L$ left invertible for all $L \geq 2$. The same can also be seen by computing $M_{0}=0_{2 \times 1}, M_{1}=0_{4 \times 2}$, and

$$
M_{2}=\left[\begin{array}{ccc}
0 & 0 & 0 \\
0 & 0 & 0 \\
0 & 0 & 0 \\
0 & 0 & 0 \\
\gamma_{12} \alpha_{21} \beta & 0 & 0 \\
\gamma_{22} \alpha_{21} \beta & 0 & 0
\end{array}\right] .
$$

This shows that generically $\operatorname{rank} M_{2}=1$, which is equal to $p+\operatorname{rank} M_{1}$ since $p=1$ and $\operatorname{rank} M_{1}=0$.

\section{NON-SCALAR UNKNOWN INPUT}

In the general case where the input is not scalar, finding a graphical characterization of delay- $L$ left invertibility is an open problem. In this section, we summarize the partial results that are known in the literature, and we present an example which contradicts a natural naive conjecture.

If one considers left invertibility, without asking for a specific delay $L$, the graphical characterization is wellknown. It involves the notion of linking: in a digraph, given two disjoint subsets of vertices $V_{1}$ and $V_{2}$, a linking from $V_{1}$ to $V_{2}$ is a collection of vertex-disjoint paths, each originated in a vertex in $V_{1}$ and ending in a vertex in $V_{2}$; the size of the linking is the number of paths.

Proposition 2 ([14, Theorem 2]): A structured system is generically left invertible if and only if there exists a linking of size $p$ from $U$ to $Y$ in $\mathcal{G}$. Moreover, if there is no such linking, then the structured system is not left invertible, for any choice of the parameters.

In the particular case of scalar input $(p=1)$, a linking of size $p$ from $U$ to $Y$ is simply a path from $u$ to $Y$. Hence, if we particularize Proposition 2 to the case $p=1$, we obtain Corollary 1.

By looking at Theorem 1 and Proposition 2 it is tempting to conjecture that generic delay- $L$ left invertibility can be characterized by the existence of a linking of size $p$ from $U$ to $Y$ in $\mathcal{G}$, such that each path in the linking has length at most $L+1$. Indeed, in Theorem 1, the condition $L \geq \operatorname{dist}(u, Y)-1$ can be rephrased as 'there exists a path

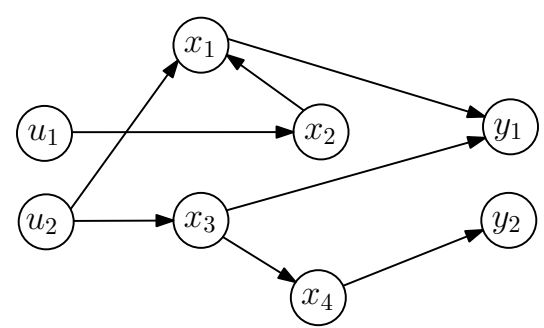

Fig. 3. Digraph $\mathcal{G}$ of the structured system in Example 2.

of length at most $L+1$ from $u$ to $Y^{\prime}$ and moreover, since $p=1$, a path from $u$ to $Y$ is a linking of size $p$ from $U$ to $Y$. However, this naive conjecture if false, as shown by the following example.

Example 2: Consider the structured system (1) with the following matrices:

$$
\begin{gathered}
A=\left[\begin{array}{cccc}
0 & \alpha_{12} & 0 & 0 \\
0 & 0 & 0 & 0 \\
0 & 0 & 0 & 0 \\
0 & 0 & \alpha_{43} & 0
\end{array}\right], \quad B=\left[\begin{array}{cc}
0 & \beta_{12} \\
\beta_{21} & 0 \\
0 & \beta_{32} \\
0 & 0
\end{array}\right], \\
C=\left[\begin{array}{cccc}
\gamma_{11} & 0 & \gamma_{13} & 0 \\
0 & 0 & 0 & \gamma_{24}
\end{array}\right], \quad D=\left[\begin{array}{ll}
0 & 0 \\
0 & 0
\end{array}\right] .
\end{gathered}
$$

Its digraph $\mathcal{G}$ is shown in Figure 3. The two paths $u_{1}, x_{2}, x_{1}, y_{1}$ and $u_{2}, x_{3}, x_{4}, y_{2}$ form a linking of size $2=p$ from $U$ to $Y$. Moreover, both paths have length 3. Since this linking has size $p$, by Proposition 2 the system is generically left invertible. However, despite both paths having length 3 , the system is not generically delay-2 left invertible. This can be seen by explicitly computing $M_{1}$ and $M_{2}$ :

$$
\begin{gathered}
M_{1}=\left[\begin{array}{ccccc}
0 & 0 & 0 & 0 \\
0 & 0 & 0 & 0 \\
0 & \gamma_{11} \beta_{12}+\gamma_{13} \beta_{32} & 0 & 0 \\
0 & 0 & 0 & 0
\end{array}\right], \\
M_{2}=\left[\begin{array}{ccccccc}
0 & 0 & 0 & 0 & 0 & 0 \\
0 & 0 & 0 & 0 & 0 & 0 \\
0 & \gamma_{11} \beta_{12}+\gamma_{13} \beta_{32} & 0 & 0 & 0 & 0 \\
0 & 0 & 0 & 0 & 0 & 0 \\
\gamma_{11} \alpha_{12} \beta_{21} & 0 & 0 & \gamma_{11} \beta_{12}+\gamma_{13} \beta_{32} & 0 & 0 \\
0 & \gamma_{24} \alpha_{43} \beta_{32} & 0 & 0 & 0 & 0
\end{array}\right],
\end{gathered}
$$

from which it is clear that generically $\operatorname{rank} M_{1}=1$ and $\operatorname{rank} M_{2}=2$, so that generically $\operatorname{rank} M_{2}<p+\operatorname{rank} M_{1}$. This shows that generically this system is not delay-2 left invertible.

The graphical characterization of delay- $L$ left invertibility has been solved in the literature for $L=0$ and $L=1$, as summarized below. Its characterization for $L \geq 2$ remains an open problem.

For the case $L=0$, by Proposition 1, delay- 0 observability is simply equivalent to $\operatorname{rank} D=p$. The generic rank of a matrix containing only zeros and distinct free parameters can be characterized as the size of the maximum matching (i.e., the largest number of vertex-disjoint edges) in a suitable 


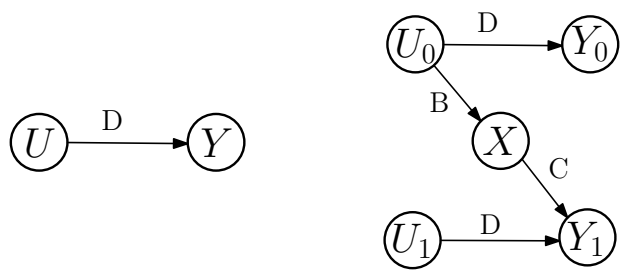

Fig. 4. Pictorial reminder of the construction of digraphs $\mathcal{K}_{0}$ (left) and $\mathcal{K}_{1}$ (right) used in Propositions 3 and 4 to characterize generic delay- 0 and delay-1 left invertibility.

bipartite graph, see e.g. Chapter 2 of the book [2]. For the matrix $D$, the relevant graph is $\mathcal{K}_{0}$, defined as the subgraph of $\mathcal{G}$ obtained by removing the state vertices (i.e., removing $X$ ); a sketch of $\mathcal{K}_{0}$ is in the left side of Figure 4 . From this classical result, we immediately obtain the following.

Proposition 3: A structured system is generically delay-0 left invertible if and only if there exists a matching of size $p$ in $\mathcal{K}_{0}$. Moreover, if there is no such matching, then the structured system is not delay-0 left invertible, for any choice of the parameters.

The case $L=1$ has been studied in [9], where a graphical characterization of delay-1 left invertibility has been obtained, involving a digraph $\mathcal{K}_{1}$ defined as follows. The vertex set contains two copies $U_{0}=\left\{u_{1}(0), \ldots, u_{p}(0)\right\}$ and $U_{1}=$ $\left\{u_{1}(1), \ldots, u_{p}(1)\right\}$ of the input vertex set $U$, two copies $Y_{0}=\left\{y_{1}(0), \ldots, y_{m}(0)\right\}$ and $Y_{1}=\left\{y_{1}(1), \ldots, y_{m}(1)\right\}$ of the output vertex set $Y$, and the state vertex set $X$. Edges are associated with the non-zero positions of $B, C, D$, as follows: there is an edge $\left(u_{j}(0), x_{i}\right)$ if and only if $B_{i j}$ is not fixed to 0 , there is an edge $\left(x_{j}, y_{i}(1)\right)$ if and only if $C_{i j}$ is not fixed to 0 , and there are edges $\left(u_{j}(0), y_{i}(0)\right)$ and $\left(u_{j}(1), y_{i}(1)\right)$ if and only if $D_{i j}$ is not fixed to 0 . See the right sketch in Figure 4 for a pictorial representation of this construction. The characterization of generic delay-1 left invertibility is the following.

Proposition 4 ([9, Theorem 2]): A structured system is generically delay-1 left invertible if and only if in $\mathcal{K}_{1}$ there exists a linking from $U_{0} \cup U_{1}$ to $Y_{0} \cup Y_{1}$ of size $p+r$, where $r$ is the size of the maximum matching in $\mathcal{K}_{0}$.

As a final remark, notice that in Theorem 1 (about delay- $L$ left invertibility for systems with scalar input), Proposition 2 (about left invertibility) and Proposition 3 (about delay-0 left invertibility), when the graphical condition fails, then the corresponding property is false for any choice of the parameters. In other words, as soon as there is at least one choice of parameters such that the property is true, then the property is true generically. However, the same does not happen for delay- $L$ left invertibility, for $L \geq 1$ and nonscalar input. Indeed, for some systems such that generically rank $M_{L}<p+\operatorname{rank} M_{L-1}$, there might exist particular parameters such that $\operatorname{rank} M_{L}=p+\operatorname{rank} M_{L-1}$; clearly such particular parameters must be such that $M_{L-1}$ has a rank which is smaller than its generic rank. An explicit example of such phenomenon happens in Example 2.
Example 2 (continued): Consider the structured system from Example 2. As shown above, for almost all parameters this system is not delay-2 left invertible: $\operatorname{rank} M_{2}<p+\operatorname{rank} M_{1}$ holds true generically. However, this system is delay-2 left invertible for some particular parameters. Indeed, if we choose $\gamma_{11}, \gamma_{13}, \beta_{12}$ and $\beta_{32}$ such that $\gamma_{11} \beta_{12}+\gamma_{13} \beta_{32}=0$ and we set all other parameters to arbitrary non-zero values, we obtain $\operatorname{rank} M_{1}=0$ and $\operatorname{rank} M_{2}=2$, thus showing that with these parameters the system is delay-2 left invertible.

\section{Conclusion}

In this paper, we have studied delay- $L$ left invertibility of structured systems with unknown input. As a main result, under the assumption that the input is scalar, we have obtained a graphical characterization of generic delay- $L$ left invertibility. Then, we have discussed the general case where the input is not scalar: we have presented the partial results present in the literature and an example disproving a natural naive conjecture. The characterization of delay- $L$ left invertibility for $L \geq 2$ remains as an open problem.

\section{REFERENCES}

[1] C.-T. Lin, "Structural controllability," IEEE Transactions on Automatic Control, vol. AC-19, no. 3, pp. 201-208, June 1974.

[2] K. Murota, Matrices and Matroids for Systems Analysis. Springer, 2000, vol. 20.

[3] J.-M. Dion, C. Commault, and J. van der Woude, "Generic properties and control of linear structured systems: a survey," Automatica, vol. 39, no. 7, pp. 1125-1144, 2003.

[4] F. Pasqualetti, F. Dörfler, and F. Bullo, "Attack detection and identification in cyber-physical systems," IEEE Transactions on Automatic Control, vol. 58, no. 11, pp. 2715-2729, Nov 2013.

[5] S. Weerakkody, X. Liu, S. H. Son, and B. Sinopoli, "A graph-theoretic characterization of perfect attackability for secure design of distributed control systems," IEEE Transactions on Control of Network Systems, vol. 4, no. 1, pp. 60-70, March 2017.

[6] Y. Mo and B. Sinopoli, "False data injection attacks in control systems," in Proc. 1st Workshop Secure Control Syst., Stockholm, Sweden, 2010.

[7] T. Boukhobza, F. Hamelin, and S. Martinez-Martinez, "State and input observability for structured linear systems: A graph-theoretic approach," Automatica, vol. 43, no. 7, pp. 1204-1210, 2007.

[8] S. Weerakkody, X. Liu, and B. Sinopoli, "Robust structural analysis and design of distributed control systems to prevent zero dynamics attacks," in 56th IEEE Conference on Decision and Control (CDC), Melbourne, Australia, Dec 2017, pp. 1356-1361.

[9] F. Garin, "Structural delay-1 input-and-state observability," in 56th IEEE Conference on Decision and Control (CDC), no. 4, Melbourne, Australia, Dec 2017, pp. 2324-2329.

[10] S. Sundaram and C. N. Hadjicostis, "Delayed observers for linear systems with unknown inputs," IEEE Trans. Automatic Control, vol. 52, no. 2, pp. 334-339, 2007.

[11] J. Massey and M. Sain, "Inverses of linear sequential circuits," IEEE Trans. Computers, vol. C17, no. 4, pp. 330-337, 1968.

[12] M. Sain and J. Massey, "Invertibility of linear time-invariant dynamical systems," IEEE Trans. Automatic Control, vol. 14, no. 2, pp. 141-149, 1969.

[13] S. Gracy, "Input and State Observability of Linear Network Systems with Application to Security of Cyber Physical Systems," Ph.D. dissertation, Université Grenoble Alpes, Nov. 2018. [Online]. Available: https://tel.archives-ouvertes.fr/tel-02047900

[14] J. W. van der Woude, "A graph-theoretic characterization for the rank of the transfer matrix of a structured system," Mathematics of Control, Signals and Systems, vol. 4, no. 1, pp. 33-40, 1991. 